\title{
Case study: flicker transfer coefficient and frequency components
}

\author{
A. Lazkano ${ }^{1}$, J.J. Gutierrez ${ }^{1}$, L.A. Leturiondo ${ }^{1}$, F. Pazos ${ }^{2}$, J. Ruiz ${ }^{1}$ \\ ${ }^{1}$ Department of Electronic and Telecommunication \\ Faculty of Engineering \\ University of the Basque Country \\ Alameda Urquijo s/n, 48013 Bilbao (Spain) \\ Phone.: +34 946017383, e-mail: andoni.lazkano@ehu.es, josejulio.gutierrez@ehu.es, luisalberto.leturiondo@ehu.es, \\ jesusmaria.ruiz@ehu.es \\ ${ }^{2}$ Iberdrola Distribución S.A. \\ Avenida San Adrián, 48 \\ 48003 Bilbao (Spain) \\ Phone.: +34 944151 411, e-mail: fj.pazos@iberdrola.es
}

\begin{abstract}
Voltage fluctuations related to light flicker can propagate along a distribution network with their attenuation being affected by the topology of the network, the line impedances, and the characteristics of the loads and the transformers. The propagation leads to different simultaneous flicker measurements at different voltage levels. This work documents a case study in which three points in a distribution network were monitored, with the flicker level being measured simultaneously at $132 \mathrm{KV}, 30 \mathrm{KV}$, and $13 \mathrm{KV}$. Two types of flicker transfer coefficient are discussed, taking into account the impact of different types of loads, the spectral characteristics involved in the flicker measurements, and possible strategies for flagging dip events at the three voltage levels.
\end{abstract}

\section{Key words}

Power quality, flicker measurement, flicker transfer coefficient, frequency component, dip event.

\section{Introduction}

Light flicker is a perception problem and consequently it is difficult to quantify. The IEC flickermeter quantifies the physiological annoyance suffered by the human eye when subjected to the luminous fluctuation of a reference incandescent lamp. The IEC 61000-4-15 standard establishes the functional and design specifications for the IEC flickermeter [1]. This standard defines Pst, the 10-minute short-term flicker severity, and Plt, the 2-hour long-term flicker severity, as parameters for assessing the annoyance produced by light fluctuations.

Distribution utilities have an increasing level of interest in power-quality monitoring and in meeting limits stipulated by regulatory entities. Some companies report significant flicker levels at various points in their distribution systems with no associated customer complaints. The compatibility levels are being questioned and the flicker objectives for power systems are being reviewed by the CIGRE/CIRED Joint Task Force C4.1.08 "Review of Flicker Objectives of HV, MV and LV systems.” This paper is a response to the proposed scope of the task force that documents one experience of measuring flicker simultaneously at three different voltage levels $(132 \mathrm{KV}, 30 \mathrm{KV}$ and $13 \mathrm{KV})$. The three measuring points were selected at a location in the north of Spain, with several iron and steel plants nearby. A flicker severity level $\mathrm{Plt}_{95 \%}=1.32$ was measured on the $\mathrm{HV}$ (High Voltage) system during the monitoring experience. There was no customer complaint associated with such flicker levels.

This study takes into consideration some key technical issues. The theory of flicker propagation in power systems is documented in [2]-[3]. Flicker transfer coefficients were evaluated using the trend lines of Pst scatter plots at the different voltage levels. The statistical measurements Pst $_{95 \%}$, Pst ${ }_{99 \%}$, and $\mathrm{Plt}_{95 \%}$ were also used to characterize the flicker transfer coefficients. The flagging of dip events [4] has been implemented, following various strategies to achieve consistency in the flicker transfer coefficients for the different measurements.

Finally, we have investigated the possibility of attributing the flicker transfer coefficients' characteristics to a particular frequency range. A flicker measurement scheme based on multiband frequency analysis is proposed and is applied to the measuring points. The percentage of the total Pst contained in each band is calculated. 


\section{Measuring System and Location}

The data acquisition system used to monitor the flicker indexes is presented. In addition, the topology and conditions of the HV and MV (Medium Voltage) locations are described.

\section{A. Flicker Measuring System}

The flicker measurement system comprises a registering module and an off-line signal-processing module. The second module implements the IEC 61000-4-15 standard in MATLAB. This flickermeter is a complete digital implementation previously used in other studies [5].

The registering module (see Fig. 1) is a hardware and software platform comprising two subsystems:

1) A conditioning system to transform the threephase voltage and current levels to the appropriate levels for processing. This hardware platform provides four voltage channels and four current channels. Different scales are provided per channel. Each channel includes a fifth-order Butterworth antialiasing filter with adjustable cutoff frequency.

2) The registering software, which runs on a PC laptop and controls the signal acquisition using a 12-bit resolution A/D card. This software selects the parameters that configure the register: sampling frequency up to 25600 samples per second; channels to be recorded; scale of each selected voltage and current channel; and duration of the register.

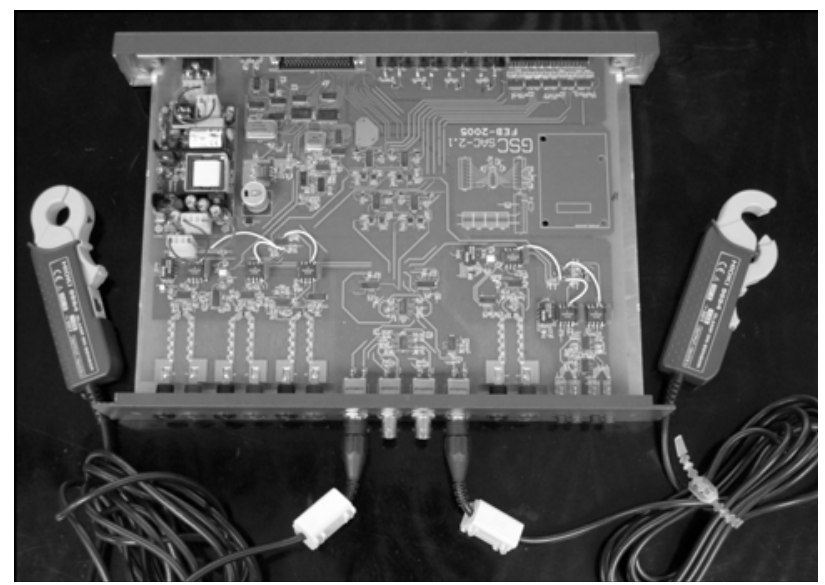

Fig. 1. Flicker Measurement System: Registering Module.

\section{B. Measuring Location}

Fig. 2 shows the three synchronized recording systems connected at A (132KV), B (30KV) and C (13KV). The flicker source was upstream, at $220 \mathrm{KV}$, and was identified as an electric arc furnace that, nevertheless, mitigated the perturbations via a Static Var Compensator.

Three-phase measurements were recorded at each voltage level, using a sampling frequency of $6400 \mathrm{~S} / \mathrm{s}$.
Within the area, the industrial loads were mainly connected at the $30 \mathrm{KV}$ level in the form of a considerably meshed network. The residential loads were mainly connected at the $13 \mathrm{KV}$ level via radial connections. There were no flicker complaints in the related area.

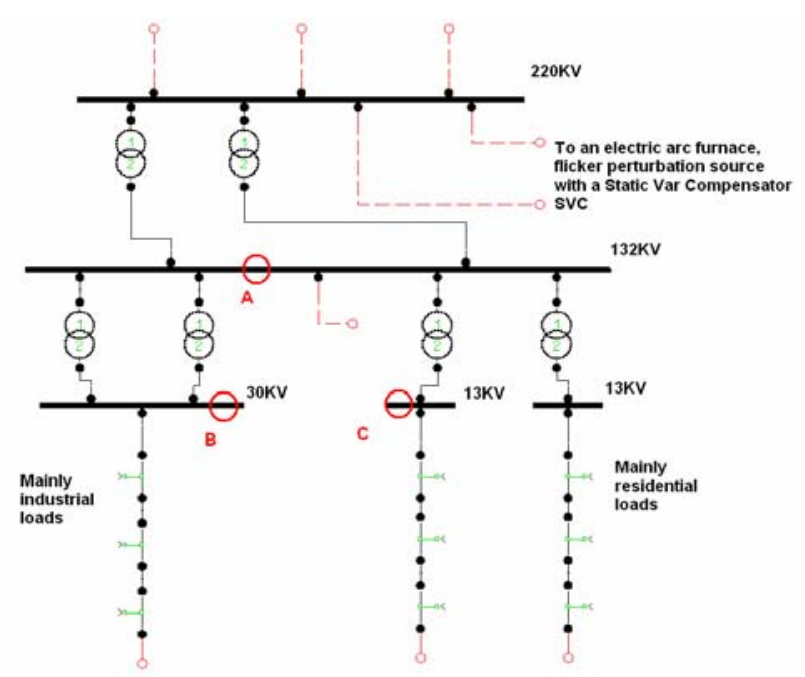

Fig. 2. Network topology of the studied location.

\section{Flicker Measurements and Transfer Coefficients}

The short-term flicker severity values were calculated for the three locations. The evolution of Pst at the $132 \mathrm{KV}$ level was selected as the reference value. Fig. 3 shows this evolution of Pst at the 132KV level.

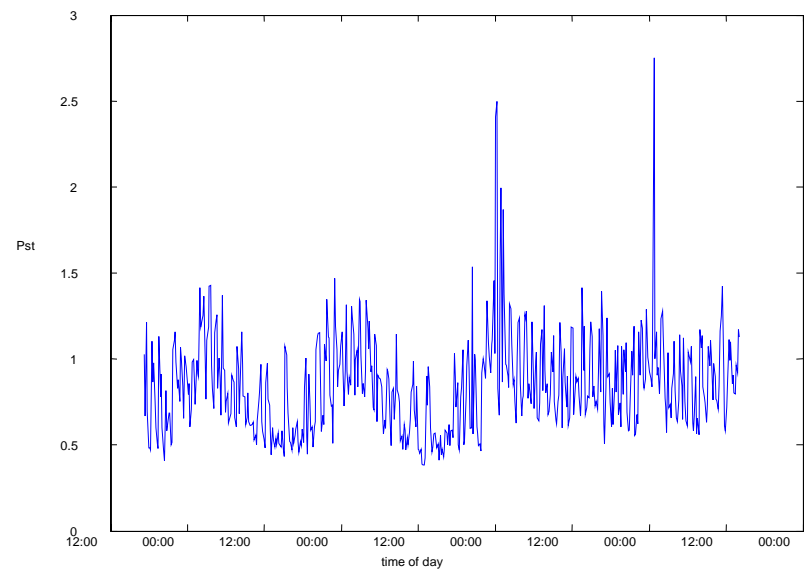

Fig. 3. Evolution of the Pst at 132KV HV level.

The flicker measurements for each location are presented in Table I, showing the 95\% and 99\% cumulative probability values of Pst (Pst ${ }_{95 \%}$ and $\mathrm{Pst}_{99 \%}$ ) and the 95\% cumulative probability values of Plt (Plt $\left.{ }_{95} \%\right)$. Despite the flicker indexes being noticeable in the residential locations, it is remarkable that no certified flicker complaints were received. The flicker indexes were, unsurprisingly, considerable at the $132 \mathrm{KV}$ level. However, flicker indexes were lower at the voltage level related to industrial loads. 
TABLE I. - Flicker indexes measured at each voltage level.

\begin{tabular}{|l|c|c|c|}
\hline & Pst $_{95 \%}$ & Pst $_{99 \%}$ & Plt $_{95 \%}$ \\
\hline Point A - 132KV & 1.2904 & 1.5695 & 1.3278 \\
\hline Point B - 30KV & 0.9294 & 1.3379 & 0.8560 \\
\hline Point C - 13KV & 1.2492 & 1.4767 & 1.1790 \\
\hline
\end{tabular}

Table II shows the calculated flicker transfer coefficients from the $132 \mathrm{KV}$ level to the $30 \mathrm{KV}$ level and the $13 \mathrm{KV}$ level in terms of the Pst $_{95 \%}$, Pst ${ }_{99 \%}$ and $\mathrm{Plt}_{95 \%}$ values.

TABLE II. - Flicker transfer coefficients (TCs).

\begin{tabular}{|l|c|c|c|}
\hline & Pst $_{95 \%}$ & Pst $_{99 \%}$ & Plt $_{95 \%}$ \\
\hline TC $_{132 / 30}$ & 0.720 & 0.852 & 0.648 \\
\hline TC $_{132 / 13}$ & 0.968 & 0.941 & 0.888 \\
\hline
\end{tabular}

The transfer coefficients were also evaluated from the instantaneous Pst values. Figs. 4 and 5 show the relation between the Pst values at the $30 \mathrm{KV}$ and $13 \mathrm{KV}$ voltage levels, respectively, and the Pst values at the $132 \mathrm{KV}$ level, via scattering plots. By calculating the transfer trend line, shown as a red line in Fig. 4, it is possible to assess the transfer coefficient from $132 \mathrm{KV}$ to $30 \mathrm{KV}$ as the slope of the trend. The result is about 0.7663 . In the same way, Fig. 5 shows a transfer coefficient from $132 \mathrm{KV}$ to $13 \mathrm{KV}$ of about 0.9836 . These values are slightly higher than the values given in Table I.

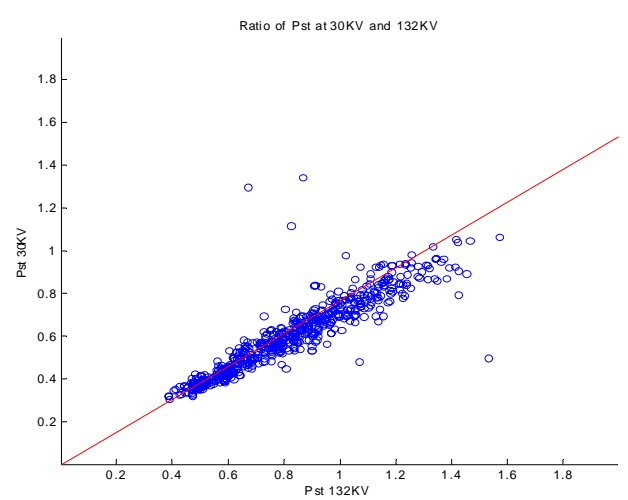

Fig. 4. Pst values at point A versus Pst values at point B.

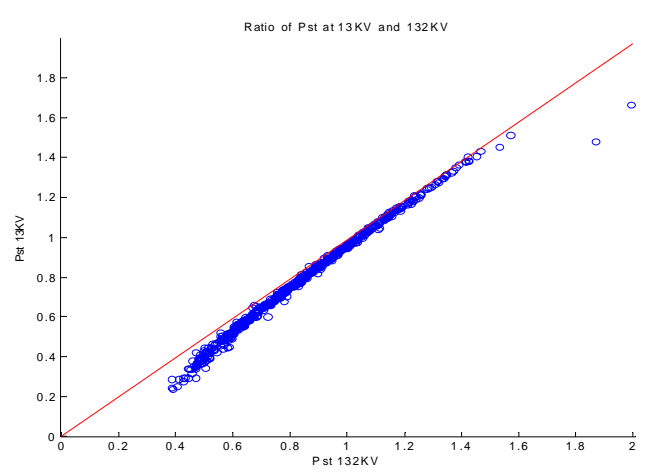

Fig. 5. Pst values at point A versus Pst values at point C.

It is interesting to analyze the relationship between the two flicker transfer coefficients and the original Pst index at $132 \mathrm{KV}$. In Fig. 6, it is shown that the transfer coefficient from $132 \mathrm{KV}$ to $30 \mathrm{KV}, \mathrm{TC}_{132 / 30}$, is randomly related to Pst at the $132 \mathrm{KV}$ level. However, the transfer coefficient from $132 \mathrm{KV}$ to $13 \mathrm{KV}, \mathrm{TC}_{132 / 13}$, shows that the flicker transmission depends on the Pst index upstream. This relationship becomes constant for values of Pst greater than 0.8 at $132 \mathrm{KV}$.

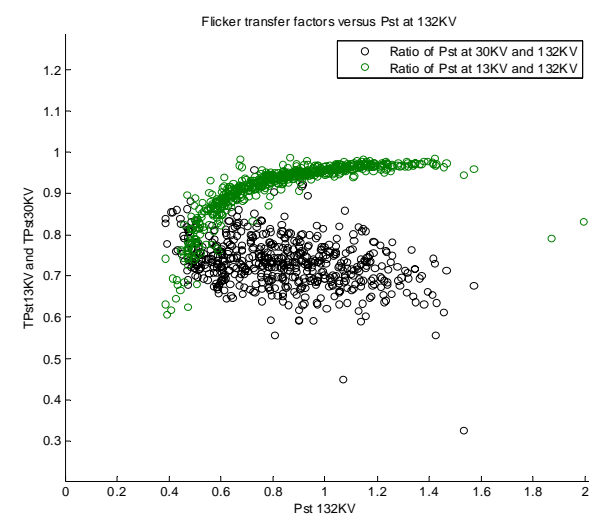

Fig. 6. Flicker transfer coefficients $\mathrm{TC}_{132 / 13}$ and $\mathrm{TC}_{132 / 30}$ versus Pst at $132 \mathrm{KV}$.

\section{Flagging of Dip Events}

The recorded signals were processed with the aim of identifying dip events. A root-mean-square (rms) value was calculated every half cycle. At the $30 \mathrm{KV}$ level, four dip events were found. However, as they did not drop by $10 \%$ of the nominal voltage at the other levels, they were not flagged as dip events at $132 \mathrm{KV}$ and $30 \mathrm{KV}$, according to IEC 61000-4-30 [6]. The same situation was identified at $132 \mathrm{KV}$, where one dip event at $132 \mathrm{KV}$ was also noticeable at the $30 \mathrm{KV}$ and $13 \mathrm{KV}$ levels. However, by remaining above $90 \%$ of the nominal voltage, it was not flagged as a dip event at either of these levels.

Fig. 7 shows one of these cases, namely a dip event at the $30 \mathrm{KV}$ level, related to a Pst peak value of 4.2 near midnight on February $17^{\text {th }}$, 2007. It is remarkable that the voltage remained above $90 \%$ of the nominal voltage at $13 \mathrm{KV}$ and $132 \mathrm{KV}$, fluctuating within a range of $+1 \%$ and $-8 \%$ respectively. Therefore, these would not be flagged as dip events.
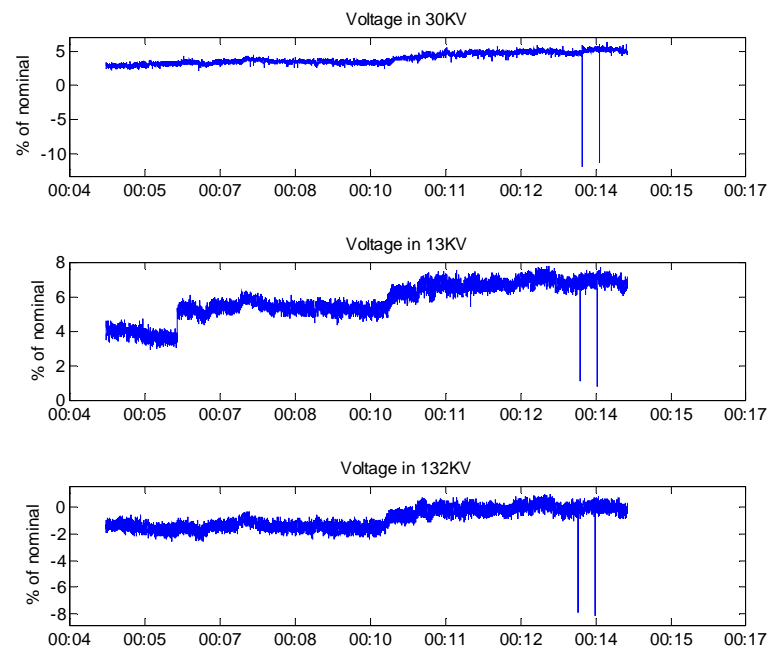

Fig. 7. Dip event at 30KV in site C: impact at sites A and B. 


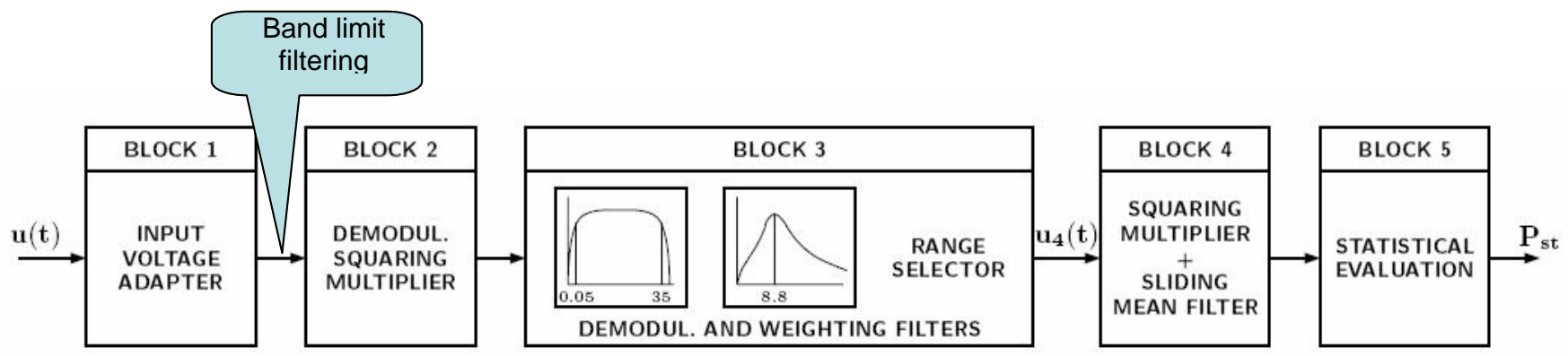

Fig. 8. Block diagram of the IEC 61000-4-15 standard for the flickermeter.

The flicker transfer coefficients changed when Pst values related to dip events were excluded from the flicker measurements. The flicker transfer coefficients calculated from the various Pst statistical indexes then became convergent.

When only the dip events related to the respective voltage levels are excluded from the flicker measurements (that is, the four Pst values at $30 \mathrm{KV}$ and the only Pst value at $132 \mathrm{KV}$ ), the conclusions are different. Table III shows the recalculated transfer coefficients for the $30 \mathrm{KV}$ and $13 \mathrm{KV}$ levels, using the Pst $_{95 \%}$, Pst ${ }_{99 \%}$, and Plt $_{95 \%}$ values. The difference between the same transfer coefficients decreases.

Differences of up to $19.32 \%$ were reported for the $132 \mathrm{KV} / 30 \mathrm{KV}$ transfer coefficients, and up to $16.8 \%$ for the $132 \mathrm{KV} / 13 \mathrm{KV}$ transfer coefficients. These results reinforce the considerations of the CIGRE/CIRED Joint Task Force C4.1.08, namely, that some measurement errors in the flicker measurements may be caused by not flagging dip events.

TABLE III. - Flicker transfer coefficients when dip events are excluded at each voltage level and the percentage difference from the no-exclusion case.

\begin{tabular}{|c|c|c|c|}
\hline & Pst $_{95 \%}$ & Pst $_{99 \%}$ & Plt $_{95 \%}$ \\
\hline TC $_{132 / 30}$ & 0.7176 & 0.6874 & 0.7308 \\
& $(-0.33 \%)$ & $(-19.32 \%)$ & $(+12.78 \%)$ \\
\hline $\mathrm{TC}_{132 / 13}$ & 0.9722 & 0.9656 & 1.0372 \\
& $(+0.43 \%)$ & $(+2.61 \%)$ & $(+16.8 \%)$ \\
\hline
\end{tabular}

\section{Band-Limited Flicker Measuring}

The influence of different frequency bands on the flicker measurement was studied by implementing a multiband flickermeter. Five frequency bands around $50 \mathrm{~Hz}$ were considered, and five Infinite Impulse Response (IIR) digital band-pass filters were designed. These filters were inserted in the flicker evaluation process, at the output of Block 1 in the diagram of the IEC 61000-4-15 standard (Fig. 8). In this way, it becomes possible to obtain the spectral characteristics contributing to Pst.

All IIR filters were designed using the $6^{\text {th }}$ order Cauer approximation, defining a maximum attenuation of $0.1 \mathrm{~dB}$ for the pass band and a minimum attenuation of $60 \mathrm{~dB}$ for the stop band.
The Pst percentage contained in each band was calculated. Table IV shows the mean values of the percentages calculated for all the measurements at the three voltage levels. For each band, the differences between the mean values at each voltage level were always low. It was evident that the percentage increases progressively as the band was widened. It is possible to infer that the 40-62.5 $\mathrm{Hz}$ band contains nearly all the flicker content of the input signal.

TABLE IV. - Percentage of the total Pst contained in each frequency band.

\begin{tabular}{|c|c|c|c|c|}
\hline & Frequencies & $13 \mathrm{KV}$ & $30 \mathrm{KV}$ & $132 \mathrm{KV}$ \\
\hline Band1 & $49-51.02 \mathrm{~Hz}$ & $22.55 \%$ & $21.87 \%$ & $20.01 \%$ \\
\hline Band2 & $47.5-52.6 \mathrm{~Hz}$ & $42.61 \%$ & $40.19 \%$ & $38.73 \%$ \\
\hline Band3 & $45-55.55 \mathrm{~Hz}$ & $74.44 \%$ & $70.77 \%$ & $71.28 \%$ \\
\hline Band4 & $40-62.5 \mathrm{~Hz}$ & $99.24 \%$ & $96.77 \%$ & $97.13 \%$ \\
\hline Band5 & $35-71.42 \mathrm{~Hz}$ & $100 \%$ & $99.41 \%$ & $99.66 \%$ \\
\hline
\end{tabular}

Finally, the spectral analysis of the components contributing to flicker was completed by processing the output of Block $3, u_{4}(t)$, in the diagram of the IEC 610004-15 standard shown in Fig. 8. This signal represents the weighted envelope of the input signal $u(t)$. The normalized power spectral density of $u_{4}(t)$ was calculated for each voltage level. Fig. 9 shows the values for the frequency band of the envelope that influences flicker, namely $0-35 \mathrm{~Hz}$ of $u_{4}(t)$.

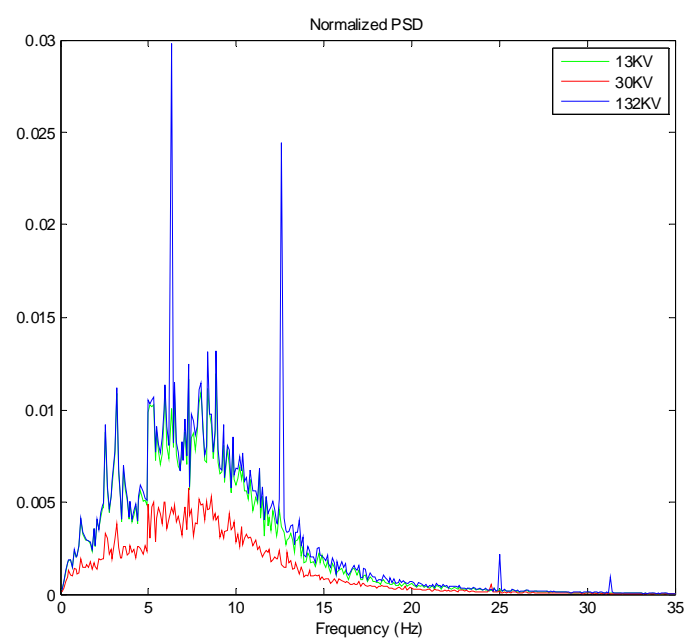

Fig. 9. Normalized power spectral density of $u_{4}(t)$ at each monitored voltage level. 
The weighted envelope at the $30 \mathrm{KV}$ voltage level, related mainly to industrial loads, is below those at $13 \mathrm{KV}$ and $132 \mathrm{KV}$. Nevertheless, it is not possible to attribute the reduction in Pst to any particular frequency of the input signal. The global difference between the power spectral densities at $132 \mathrm{KV}$ and $30 \mathrm{KV}$ explains the reduction in the Pst values.

\section{Conclusions}

We carried out three synchronized flicker measurements at $132 \mathrm{KV}, 30 \mathrm{KV}$, and $13 \mathrm{KV}$ voltage levels using three recording systems. The results of the measurement are presented, showing different transfer coefficient values. The reasons for the dissimilar ratios can be identified as differences in the network structure (meshed vs. radial) and different types of loads (industrial with induction motors vs. mainly residential). Other inconsistencies are noticed between transfer coefficients that depend on the flicker statistics selected for evaluating the ratios.

These inconsistencies can be reduced by flagging dip events. However, excluding voltage dips $>10 \%$ of the nominal voltage according to IEC 61000-4-30 is not sufficient, because smaller voltage drops can create high flicker values when there are dip events at adjacent voltage levels.

We studied the contribution of different frequency bands to the flicker measurements. A band-limited flickermeter was proposed for this purpose. The flicker spectral characteristics were investigated at the different voltage levels by means of a spectral analysis of the weighted envelope of the input signal to the flickermeter.

\section{Acknowledgment}

The financial support from the "Ministerio de Educación y Ciencia” of Spain, DPI2006-15083-C02-02 Project, is gratefully acknowledged.

\section{References}

[1] IEC std., 61000-4-15+A1, Electromagnetic Compatibility (EMC) Part 4: Testing and Measurement Techniques Section 15: Flickermeter Functional and Design Specifications, 2003.

[2] S. Perera, D. Robinson, S. Elphick, D. Geddey, N. Browne, V. Smith and V. Gosbell,, "Synchronized Flicker Measurement for Flicker Transfer Evaluation in Power Systems”, IEEE Trans. On Power Delivery, Vol. 21, No. 3, pp 1477-1482, July 2006

[3] B. Novo Ramos, J. F. Manzanedo Garcia and M. Perez Donsicin, "Study of Flicker Generation and Transmission in Interconnected Electric Grids", 10th International Conference on Harmonics and Quality of Power. Volume 1, pp: 66-70, 6-9 Oct. 2002.

[4] G. Bucci, E. Fiorucci and C. Landi, "Digital Measurement Equipment for Steady-State PQ Measurements", IEEE Bologna PowerTech Conference, Volume 4, pp: 6, 23-26 June 2003.

[5] J.J. Gutierrez, J. Ruiz, and S.R. de Gauna, "Linearity of the IEC Flickermeter Regarding Amplitude Variations of Rectangular Fluctuations," Power Delivery, IEEE Transactions on, vol. 22, no. 1, pp. 729\{731, 2007.

[6] IEC std., 61000-4-30, Electromagnetic Compatibility (EMC) Part 4: Testing and Measurement Techniques Section 30: Power Quality Measurement Methods, 2003 